\title{
ISOMETRIC HOMOTOPY IN CODIMENSION TWO
}

\author{
BY
}

\author{
JOHN DOUGLAS MOORE
}

\begin{abstract}
This article investigates the structure of the space of isometric immersions from a simply connected $n$-dimensional Riemannian manifold with positive sectional curvatures into $(n+2)$-dimensional Euclidean space $E^{n+2}$. It is proven that if $n \geqslant 4$ and $M^{n}$ is such a manifold which admits a $C^{\infty}$ isometric immersion as a hypersurface in $E^{n+1}$, then any $C^{\infty}$ isometric immersion from $M^{n}$ into $E^{n+2}$ is $C^{2 n-4}$ homotopic through isometric immersions to an immersion whose image lies in some hyperplane.
\end{abstract}

1. Introduction. According to well-known rigidity theorems, if $M^{n}$ is an $n$-dimensional compact Riemannian manifold with positive sectional curvatures $(n \geqslant 2)$, then any two isometric immersions from $M^{n}$ into $(n+1)$-dimensional Euclidean space $E^{n+1}$ differ by a rigid motion. In particular, the $n$-dimensional sphere $S^{n}$ of constant curvature one is rigid in $E^{n+1}$. Now rigidity is a very "unstable" phenomenon: $S^{n}$ is not rigid in $E^{n+2}$ because there is an infinite-dimensional family of composition isometric immersions

$$
S^{n} \subseteq E^{n+1} \rightarrow E^{n+2}
$$

where $E^{n+1}$ is isometrically immersed in $E^{n+2}$ as a cylinder. Nevertheless, Whitt [W] was able to show that some vestige of rigidity remains in codimension two; he argued that any isometric immersion from $S^{n}$ into $E^{n+2}$ is homotopic through isometric immersions to an immersion whose image lies in some hyperplane of $E^{n+2}$, when $n \geqslant 4$. Although Whitt did not establish differentiability of the isometric homotopy, Whitt's work on this topic is very important because it suggests a new viewpoint for rigidity theory in codimension greater than one.

This article will prove an extension of Whitt's theorem including differentiability up to order $2 n-4$.

THEOREM. Suppose that $n \geqslant 4$ and $k$ is an integer, $1 \leqslant k \leqslant 2 n-4$. If $M^{n}$ is a simply connected $n$-dimensional Riemannian manifold with positive sectional curvatures which possesses a $C^{k+3}$ isometric immersion into $E^{n+1}$, then any $C^{k+3}$ isometric immersion from $M^{n}$ into $E^{n+2}$ is $C^{k}$ homotopic through isometric immersions to an immersion whose image lies in some hyperplane of $E^{n+2}$.

In particular, any smooth isometric immersion from $S^{n}$ into $E^{n+2}$ is unknotted, when $n \geqslant 4$.

It is conjectured that a $C^{\infty}$ version of the above theorem is true, but although Whitt was kind enough to credit us with a proof in the $C^{\infty}$ case, we have not been able to construct such a proof. The difficulty occurs at the so-called "umbilic

Received by the editors February 27, 1984 and, in revised form, February 1, 1985.

1980 Mathematics Subject Classification. Primary 53C42. 
points", points where the second fundamental form fails to generate the entire normal space. In this regard, we call attention to Henke's important investigation [H2] regarding the possibility of extending an isometric immersion from $S^{n}$ into $E^{n+2}$ to an isometric immersion from an open subset of $E^{n+1}$ containing $S^{n}$ into $E^{n+2}$; Henke found counterexamples to local extendability near umbilic points, and his work suggests that there may be difficulties in proving smoothness of isometric homotopies near these points.

Aside from differentiability, the geometric ideas needed to prove our theorem are contained in $\S 2$. Our differentiability results are established in $\S 4$ by means of the cone lemma of $\S 3$.

The author is deeply indebted to Richard Bishop and Wolfgang Henke for pointing out errors in earlier versions of this article.

2. The main ideas of the proof. By hypothesis, $M^{n}$ possesses a $C^{k+3}$ isometric immersion into $E^{n+1}$; call this immersion $\tilde{f}$. We will show that if $f$ is an arbitrary $C^{k+3}$ isometric immersion from $M^{n}$ into $E^{n+2}$, then $f$ is $C^{k}$ homotopic through isometric immersions to $\phi \circ i \circ \tilde{f}$, where $\phi: E^{n+2} \rightarrow E^{n+2}$ is a rigid motion and $i$ : $E^{n+1} \rightarrow E^{n+2}$ is the inclusion map.

If $p \in M$, let

$$
\alpha: T_{p} M \times T_{p} M \rightarrow N_{p} M \quad \text { and } \quad \tilde{\alpha}: T_{p} M \times T_{p} M \rightarrow \tilde{N}_{p} M
$$

denote the second fundamental forms of $f$ and $\tilde{f}$ respectively, and let $W=N_{p} M \oplus$ $\tilde{N}_{p} M$. Define a symmetric bilinear map $\beta: T_{p} M \times T_{p} M \rightarrow W$ by $\beta=\alpha \oplus \tilde{\alpha}$, and use the positive definite inner products in $N_{p} M$ and $\tilde{N}_{p} M$ to define a Lorentz inner product $\langle$,$\rangle on the three-dimensional vector space W$,

$$
\langle u \oplus \tilde{u}, v \oplus \tilde{v}\rangle=\langle u, v\rangle-\langle\tilde{u}, \tilde{v}\rangle .
$$

It follows immediately from the Gauss equations for $f$ and $\tilde{f}$ that

$$
\langle\beta(x, z), \beta(y, w)\rangle-\langle\beta(x, w), \beta(y, z)\rangle=0 \text { for all } x, y, z, w \in T_{p} M,
$$

and thus in the terminology of $[\mathbf{M}], \beta$ is flat with respect to $\langle$,$\rangle .$

We claim that because of our curvature assumption on $M$, there exist orthonormal bases $\left(e_{n+1}, e_{n+2}\right)$ and $\left(\tilde{e}_{n+1}\right)$ for $N_{p} M$ and $\tilde{N}_{p} M$ respectively such that

$$
\left\langle\alpha, e_{n+1}\right\rangle=\left\langle\tilde{\alpha}, \tilde{e}_{n+1}\right\rangle \text { and }\left\langle\alpha, e_{n+2}\right\rangle \text { has rank } \leqslant 1 \text {. }
$$

Indeed, if $x \in T_{p} M$, we define $\beta(x): T_{p}(M) \rightarrow W$ by setting $\beta(x)(y)=\beta(x, y)$. Choose $x \in T_{p} M$ so that $\beta(x)\left(T_{p} M\right)$ has maximum possible dimension; then $\beta(y)\left(T_{p} M\right)$ will vary smoothly with $y$ for $y$ sufficiently close to $x$. Let

$$
N(\beta(x))=\left\{n \in T_{p} M: \beta(x)(n)=0\right\},
$$

and check that if $n \in N(\beta(x))$,

$$
\beta(y, n)=\frac{1}{t} \beta(x+t y, n) \in \lim _{t \rightarrow 0} \beta(x+t y)\left(T_{p} M\right)=\beta(x)\left(T_{p} M\right),
$$

whereas since $\beta$ is flat,

$$
\left\langle\beta(y, n), \beta(x)\left(T_{p} M\right)\right\rangle=\left\langle\beta(x, n), \beta(y)\left(T_{p} M\right)\right\rangle=0,
$$


Thus if the restriction of the Lorentz inner product $\langle$,$\rangle to \beta(x)\left(T_{p} M\right)$ were nondegenerate, it would follow that

$$
\begin{aligned}
n & \in N(\beta(x)) \Rightarrow \beta(y, n)=0 \text { for all } y \in T_{p} M \\
& \Rightarrow \text { (via Gauss equation) } R(n, y, z, w)=0 \text { for all } y, z, w \in T_{p} M,
\end{aligned}
$$

where $R$ is the Riemann-Christoffel curvature tensor of $M^{n}$. Since $n \geqslant 4$, and hence $N(\beta(x)) \neq 0$, this contradicts our hypothesis that all the sectional curvatures of $M$ are positive.

We conclude that the restriction of $\langle$,$\rangle to \beta(x)\left(T_{p} M\right)$ is degenerate, and hence there exist unit length vectors $e_{n+1} \in N_{p} M$ and $\tilde{e}_{n+1} \in \tilde{N}_{p} M$ such that $\left(e_{n+1}, \tilde{e}_{n+1}\right)$ is a null vector in $\beta(x)\left(T_{p} M\right)$ satisfying

$$
\left\langle\left(e_{n+1}, \tilde{e}_{n+1}\right), \beta(x)\left(T_{p} M\right)\right\rangle=0 .
$$

If $n \in N(\beta(x))$,

$$
\begin{aligned}
\beta(n)\left(T_{p} M\right) & \subseteq \beta(x)\left(T_{p} M\right) \Rightarrow\left\langle\left(e_{n+1}, \tilde{e}_{n+1}\right), \beta(n)\left(T_{p} M\right)\right\rangle=0 \\
& \Rightarrow\left\langle\alpha(n, y), e_{n+1}\right\rangle=\left\langle\tilde{\alpha}(n, y), \tilde{e}_{n+1}\right\rangle \text { for all } y \in T_{p} M .
\end{aligned}
$$

Now choose $e_{n+2} \in N_{p} M$ perpendicular to $e_{n+1}$, and it is straightforward to verify (1) via the Gauss equation.

Orient $M$ so that the corresponding unit normal $\tilde{e}_{n+1}$ to the immersion $\tilde{f}$ : $M \rightarrow E^{n+1}$ has the property that the symmetric bilinear form $\left\langle\tilde{\alpha}, \tilde{e}_{n+1}\right\rangle$ is positive definite. The orientation for $M$ determines an orientation of the normal bundle $N M$ of the immersion $f: M \rightarrow E^{n+2}$. Having chosen $\tilde{e}_{n+1}$, we can specify the orthonormal sections $e_{n+1}, e_{n+2}: M \rightarrow N M$ completely by requiring that $\left(e_{n+1}(p), e_{n+2}(p)\right)$ be the unique positively oriented orthonormal basis for $N_{p} M$ such that $\left(e_{n+1}(p), e_{n+2}(p)\right)$ and $\left(\tilde{e}_{n+1}(p)\right)$ satisfy condition (1). It is not difficult to check that $e_{n+1}$ and $e_{n+2}$ are continuous sections (using an argument similar to that of [H1, Satz 2]), and indeed we will demonstrate in $\S 4$ that $e_{n+1}$ and $e_{n+2}$ are $C^{k}$, a fact that we will assume for the remainder of this section.

We will need to make use of the fundamental existence and uniqueness theorem for submanifolds [BC, §10.8]. Let $\operatorname{Iso}\left(M^{n}, E^{N}\right)$ denote the equivalence classes of isometric immersions from $M^{n}$ into $E^{N}$, two isometric immersion being regarded as equivalent if they differ by a rigid motion of $E^{N}$. According to the fundamental existence and uniqueness theorem, under the assumption that $M^{n}$ is simply connected, elements of $\operatorname{Iso}\left(M, E^{N}\right)$ are in one-to-one correspondence with triples $\left(E, \nabla^{\perp}, \alpha\right)$, where $E$ is an $(N-n)$-dimensional vector bundle over $M$ with fiber metric $\langle$,$\rangle such that T M \oplus E$ is trivial, $\nabla^{\perp}$ is a metric connection in $E$, and $\alpha$ : $T M \times T M \rightarrow N M$ is a fiberwise symmetric bilinear map such that $\nabla^{\perp}$ and $\alpha$ satisfy the Cartan structure equations which will be described in the next paragraph. Under this correspondence, $E$ becomes the normal bundle of the isometric immersion, $\nabla^{\perp}$ the normal connection, and $\alpha$ the second fundamental form.

It is easiest to describe the Cartan structure equations by means of a locally defined adapted orthonormal moving frame $\left(e_{1}, \ldots, e_{n}, e_{n+1}, \ldots, e_{N}\right)$; here $\left(e_{1}, \ldots, e_{n}\right)$ are orthonormal sections of $T M$, whereas $\left(e_{n+1}, \ldots, e_{N}\right)$ are orthonormal 
sections of $E$. Our index conventions will be

$$
1 \leqslant I, J, K \leqslant N ; \quad 1 \leqslant i, j, k \leqslant n ; \quad n+1 \leqslant \lambda, \mu, \nu \leqslant N .
$$

We define one-forms $\omega_{i j}=-\omega_{j i}$ and $\omega_{\lambda \mu}=-\omega_{\mu \lambda}$ by

$$
\omega_{i j}=\left\langle e_{i}, \nabla e_{j}\right\rangle, \quad \omega_{\lambda \mu}=\left\langle e_{\lambda}, \nabla^{\perp} e_{\mu}\right\rangle,
$$

where $\nabla$ is the Levi-Civita connection in $T M, \nabla^{\perp}$ the given connection in $E$, and we define $\omega_{i \lambda}$ and $\omega_{\lambda i}$ by

$$
\omega_{\lambda i}=-\omega_{i \lambda}=\left\langle e_{\lambda}, \alpha\left(e_{i}, \cdot\right)\right\rangle .
$$

Then the Cartan structure equations can be formulated as

$$
d \omega_{I J}=-\sum_{K} \omega_{I K} \wedge \omega_{K J}
$$

Thus the problem of constructing a one-parameter family of isometric immersions $f_{t}: M^{n} \rightarrow E^{n+2}$ such that $f_{1}=f$ and $f_{0}=\phi \circ i \circ \tilde{f}$ is reduced to the equivalent problem of constructing a one-parameter family $\left(E, \nabla_{t}^{+}, \alpha_{t}\right)$ satisfying the Cartan structure equations described above, such that $\nabla_{1}^{\perp}$ and $\alpha_{1}$ are the normal connection and second fundamental form of $f$ and $\nabla_{0}^{+}$, and $\alpha_{0}$ are the normal connection and second fundamental form of $\phi \circ i \circ \tilde{f}$. Under the assumption that $f$ and $\tilde{f}$ are $C^{k+3}$, the $\nabla_{t}^{\perp}$ and $\alpha_{t}$ we construct will be $C^{k}$ and will depend in a $C^{k}$ fashion upon $t$, and the resulting homotopy $f_{t}: M^{n} \rightarrow E^{n+2}$ will also be $C^{k}$.

We now work locally, using an adapted orthonormal frame $\left(e_{1}, \ldots, e_{n}, e_{n+1}, e_{n+2}\right)$ such that $\left(e_{n+1}, e_{n+2}\right)$ are the special orthonormal sections of the normal bundle $N M$ for $f$ described before. Using this frame we define $\omega_{I J}$ by (2) and (3). Similarly, we define $\tilde{\omega}_{I J}$ by using an adapted orthonormal frame $\left(e_{1}, \ldots, e_{n}, \tilde{e}_{n+1}, \tilde{e}_{n+2}\right)$ for the isometric immersion $i \circ \tilde{f}: M^{n} \rightarrow E^{n+2}$ such that $\tilde{e}_{n+2}$ is perpendicular to $E^{n+1}$ and $\tilde{e}_{n+1}$ is the unit normal to $M^{n}$ in $E^{n+1}$ distinguished by the orientation. Then $\tilde{\omega}_{i j}=\omega_{i j}$ and $\tilde{\omega}_{n+2, J}=0$. Moreover, it follows from (1) that

$$
\omega_{n+1, i}=\tilde{\omega}_{n+1, i}, \quad \omega_{n+2, i} \wedge \omega_{n+2, j}=0 .
$$

From (5) and the structure equations (4) we see that

$$
\begin{aligned}
-\sum \omega_{n+1, i} \wedge \omega_{i j} & =-\sum \tilde{\omega}_{n+1, i} \wedge \omega_{i j}=d \tilde{\omega}_{n+1, j}=d \omega_{n+1, j} \\
& =-\sum \omega_{n+1, i} \wedge \omega_{i j}-\omega_{n+1, n+2} \wedge \omega_{n+2, j},
\end{aligned}
$$

from which we conclude that

$$
\omega_{n+2, n+1} \wedge \omega_{n+2, j}=0 .
$$

Now we define $\omega_{I J}(t)$ by multiplying the last row and column of the matrix $\left(\omega_{I J}\right)$ by $t$ :

$$
\left(\omega_{I J}(t)\right)=\left(\begin{array}{cc:ccc} 
& & & \omega_{1, n+1} & t \omega_{1, n+2} \\
& \omega_{i j} & & \vdots & \vdots \\
& & & \omega_{n, n+1} & t \omega_{n, n+2} \\
\hdashline \omega_{n+1,1} & \cdots & \omega_{n+1, n} & 0 & t \omega_{n+1, n+2} \\
t \omega_{n+2,1} & \cdots & t \omega_{n+2, n} & t \omega_{n+2, n+1} & 0
\end{array}\right)
$$


The remarkable fact is that $\omega_{I J}(t)$ will satisfy the Cartan structure equations (4) for all $t$. Indeed, the equations

$$
d \omega_{i j}=-\sum \omega_{i k} \wedge \omega_{k j}-\omega_{i, n+1} \wedge \omega_{n+1, j}-\omega_{i, n+2} \wedge \omega_{n+2, j}
$$

and

$$
d \omega_{n+1, i}=-\sum \omega_{n+1, j} \wedge \omega_{j i}-\omega_{n+1, n+2} \wedge \omega_{n+2, i}
$$

remain unchanged by (5) and (6), while the equation

$$
d \omega_{n+2, I}=-\sum \omega_{n+2, J} \wedge \omega_{J I}
$$

is multiplied by $t$. We define $\nabla_{t}^{\perp}$ and $\alpha_{t}$ by

$$
\nabla_{t}{ }^{\perp} e_{\mu}=\sum_{\lambda} e_{\lambda} \omega_{\lambda \mu}(t), \quad \alpha_{t}\left(e_{i}, e_{j}\right)=\sum_{\lambda} e_{\lambda} \omega_{\lambda i}(t)\left(e_{j}\right) .
$$

According to the fundamental existence and uniqueness theorem, there is a $C^{k}$ one-parameter family of isometric immersions $f_{t}: M^{n} \rightarrow E^{n+2}$ such that $\nabla_{t}^{\perp}$ and $\alpha_{t}$ are the normal connection and second fundamental form of $f_{t}$. We can arrange that $f_{1}=f$, and by uniqueness there will be a Euclidean motion $\phi: E^{n+2} \rightarrow E^{n+2}$ such that $f_{0}=\phi \circ i \circ \tilde{f}$. The theorem is thus completely proven except for smoothness which will be treated in $\S \S 3$ and 4 .

3. The Cone Lemma. In this section we will prove a technical lemma needed for the proof of differentiability in $\S 4$.

Let $\operatorname{Sym}(n \times n)$ denote the vector space of real symmetric $n \times n$ matrices, and let

$$
V^{n-1}=\left\{A \in \operatorname{Sym}(n \times n): \exists B \in O(n) \text { such that } A=B J B^{-1}\right\},
$$

where $O(n)$ is the orthogonal group, and

$$
J=\frac{1}{\sqrt{n^{2}-n}}\left(\begin{array}{cccc}
1-n & 0 & \cdots & 0 \\
0 & 1 & \cdots & 0 \\
\vdots & \vdots & & \vdots \\
0 & 0 & \cdots & 1
\end{array}\right) .
$$

Note that $J$ is a trace-free matrix, and has length one in terms of the standard inner product $\langle\langle\rangle$,$\rangle on \operatorname{Sym}(n \times n)$ defined by $\langle\langle A, B\rangle\rangle=\operatorname{trace}(A B)$. We will call $V^{n-1}$ the Veronese manifold. There is a map $\phi: O(n) \rightarrow V^{n-1}$ defined by $\phi(B)=$ $B J B^{-1}$, which projects to an $O(n)$-equivariant diffeomorphism

$$
O(n) /(O(1) \times O(n-1)) \rightarrow V^{n-1}
$$

thus $V^{n-1}$ is diffeomorphic to $(n-1)$-dimensional real projective space.

When $n \geqslant 3$, the Veronese manifold $V^{n-1}$ has the important property that $V^{n-1} \cap\left(-V^{n-1}\right)=\varnothing$. Thus if we let $C V$ be the cone on $V^{n-1}$, i.e.,

$$
C V=\left\{A \in \operatorname{Sym}(n \times n): A=t v \text { for some } v \in V^{n-1}, t \in \mathbf{R}\right\},
$$

we can define a continuous map $\tau: C V \rightarrow \mathbf{R}$ by requiring that $\tau(t v)=t$, when $v \in V^{n-1}$. Note that $C V$ is an $n$-dimensional smooth submanifold of $\operatorname{Sym}(n \times n)$ except for a singularity at the origin, and $\tau$ is smooth except at the origin. 
CONe Lemma. Let $r$ be an integer, $0 \leqslant r \leqslant 2 n-3$. If $(a, b)$ is an open interval of real numbers, and $f:(a, b) \rightarrow C V \subseteq \operatorname{Sym}(n \times n)$ is a $C^{r}$ map, then $\tau \circ f$ is also $C^{r}$. Moreover, for each integer $k, 1 \leqslant k \leqslant r$, there exists a constant $C_{k}$ such that $\left(E_{k}\right) \quad\left|\left(d^{k} / d t^{k}\right)(\tau \circ f)(t)\right| \leqslant C_{k}\left|\left(d^{k} / d t^{k}\right)(f)(t)\right|, \quad t \in(a, b)$.

If $U=\{t \in(a, b) \mid f(t) \neq 0\}$, then clearly $\tau \circ f$ is $C^{r}$ on $U$, since $\tau$ is $C^{\infty}$ except at the vertex of the cone. The proof of the Cone Lemma will be based upon the following

Sublemma. With the same assumptions as in the Cone Lemma, the estimates $\left(E_{k}\right)$ hold for $t \in U, 0 \leqslant k \leqslant r$.

It is in the proof of the sublemma, given at the end of this section, that the hypothesis $r \leqslant 2 n-3$ is needed.

Proof that the Sublemma implies the Cone Lemma: We will prove the Cone Lemma by induction on $r$. To start the induction, note that the Cone Lemma is true for $r=0$ because $\tau$ is continuous and $|\tau \circ f|=|f|$.

Assume inductively that the Cone Lemma is true when $r$ is replaced by $r-1$.

If $f\left(t_{0}\right)=0$, the standard argument used to prove Taylor's theorem shows that there is a $C^{r-1}$ function $f_{1}:(a, b) \rightarrow C V$ such that $f(t)=\left(t-t_{0}\right) f_{1}(t)$, and the fact that $\tau$ is homogeneous implies that

$$
(\tau \circ f)(t)=\left(t-t_{0}\right)\left(\tau \circ f_{1}\right)(t) \text {. }
$$

By inductive assumption, we can differentiate $\tau \circ f$ and $\tau \circ f_{1} r-1$ times, and it follows from the Leibniz rule that

$$
(\tau \circ f)^{(r-1)}(t)=(r-1)\left(\tau \circ f_{1}\right)^{(r-2)}(t)+\left(t-t_{0}\right)\left(\tau \circ f_{1}\right)^{(r-1)}(t),
$$

where the exponent in parentheses denotes differentiation. In particular,

$$
(\tau \circ f)^{(r-1)}\left(t_{0}\right)=(r-1)\left(\tau \circ f_{1}\right)^{(r-2)}\left(t_{0}\right) .
$$

It follows from (7) that

$$
\begin{gathered}
\frac{(\tau \circ f)^{(r-1)}(t)-(\tau \circ f)^{(r-1)}\left(t_{0}\right)}{t-t_{0}}=\frac{(\tau \circ f)^{(r-1)}(t)-(r-1)\left(\tau \circ f_{1}\right)^{(r-2)}\left(t_{0}\right)}{t-t_{0}} \\
=\frac{(r-1)\left(\tau \circ f_{1}\right)^{(r-2)}(t)-(r-1)\left(\tau \circ f_{1}\right)^{(r-2)}\left(t_{0}\right)}{t-t_{0}}+\left(\tau \circ f_{1}\right)^{(r-1)}(t),
\end{gathered}
$$

which approaches $r\left(\tau \circ f_{1}\right)^{(r-1)}\left(t_{0}\right)$ as $t$ approaches $t_{0}$, because $\tau \circ f_{1}$ is $C^{r-1}$. Hence the $r$ th derivative of $\tau \circ f$ at $t_{0}$ exists and is given by the formula

$$
(\tau \circ f)^{(r)}\left(t_{0}\right)=r\left(\tau \circ f_{1}\right)^{(r-1)}\left(t_{0}\right) \text {. }
$$

Thus $(\tau \circ f)^{(r)}(t)$ exists for every $t \in(a, b)$.

It remains to show that if $t_{0} \in(a, b),(\tau \circ f)^{(r)}(t)$ is continuous for $t$ in some neighborhood of $t_{0}$, and the estimate $\left(E_{r}\right)$ holds on this neighborhood. It will be convenient to divide the discussion into two cases, depending on whether $t_{0} \in U_{r}$ or $t_{0} \in F_{r}$, where

$$
U_{r}=\left\{t \in(a, b): f^{(k)}(t) \neq 0 \text { for some } k, 0 \leqslant k \leqslant r\right\}, \quad F_{r}=(a, b)-U_{r} .
$$


Case I: $t_{0} \in U_{r}$. By the Sublemma, we can assume that $f\left(t_{0}\right)=0$, so there is a smooth function $f_{1}:(a, b) \rightarrow C V$ such that $f(t)=\left(t-t_{0}\right) f_{1}(t)$. Since some derivative of $f$ does not vanish at $t_{0}$, there is an $\varepsilon>0$ such that

$$
0<\left|t-t_{0}\right|<\varepsilon \Rightarrow f(t) \neq 0 \& f_{1}(t) \neq 0 \text {. }
$$

By the Sublemma again, $f_{1}$ is $C^{r}$ on $\left\{t: 0<\left|t-t_{0}\right|<\varepsilon\right\}$ and satisfies the estimate $\left(E_{r}\right)$ on this set, and hence

$$
\left|\left(t-t_{0}\right)\left(\tau \circ f_{1}\right)^{(r)}(t)\right| \leqslant C_{r}\left|t-t_{0}\right|\left|f_{1}^{(r)}(t)\right| \rightarrow 0
$$

as $t \rightarrow t_{0}$. Now we apply the Leibniz rule,

$$
(\tau \circ f)^{(r)}(t)=r\left(\tau \circ f_{1}\right)^{(r-1)}(t)+\left(t-t_{0}\right)\left(\tau \circ f_{1}\right)^{(r)}(t)
$$

for $0<\left|t-t_{0}\right|<\varepsilon$, to conclude that

$$
(\tau \circ f)^{(r)}(t) \rightarrow r\left(\tau \circ f_{1}\right)^{(r-1)}\left(t_{0}\right)=(\tau \circ f)^{(r)}\left(t_{0}\right)
$$

as $t \rightarrow t_{0}$, so continuity is established in this case. Moreover, by continuity, the estimate $\left(E_{r}\right)$ holds on a neighborhood of $t_{0}$.

Case II: $t_{0} \in F_{r}$. In this case it follows from the estimate $\left(E_{r-1}\right)$ that

$$
\left|\frac{(\tau \circ f)^{(r-1)}(t)-(\tau \circ f)^{(r-1)}\left(t_{0}\right)}{t-t_{0}}\right| \leqslant C_{r-1}\left|\frac{f^{(r-1)}(t)-f^{(r-1)}\left(t_{0}\right)}{t-t_{0}}\right| \rightarrow 0
$$

as $t \rightarrow t_{0}$, and hence the $r$ th derivative of $\tau \circ f$ vanishes at $t_{0}$. Thus $(\tau \circ f)^{(r)} \equiv 0$ on $F_{r}$ and the estimate $\left(E_{r}\right)$ holds on the entire interval $(a, b)$. If $t_{0} \in F_{r}$,

$$
\left|(\tau \circ f)^{(r)}(t)\right| \leqslant C_{r}\left|f^{(r)}(t)\right| \rightarrow 0
$$

as $t \rightarrow t_{0}$, so $(\tau \circ f)^{(r)}$ is continuous at $t_{0}$. This finishes the inductive step. Q.E.D.

Proof of The Sublemma. By continuity of derivatives on $U$, it will suffice to establish the estimates $\left(E_{r}\right)$ on a dense open subset of $U$, and indeed we will establish these estimates on the dense open subset $R$ of regular points, where $R$ will be specified below.

It will be convenient to change notation slightly, by writing $\tau$ in place of $\tau \circ f$, and setting $f=\tau g$, where $g: U \rightarrow V^{n-1} \subseteq \operatorname{Sym}(n \times n)$. If $x, y \in \mathbf{R}^{n}$, say $x=\left(x_{1}, \ldots, x_{n}\right)$ and $y=\left(y_{1}, \ldots, y_{n}\right)$, we let

$$
x \circ y=\left(\begin{array}{ccc}
x_{1} y_{1} & 1 / 2\left(x_{1} y_{2}+x_{2} y_{1}\right) & \cdots \\
1 / 2\left(x_{2} y_{1}+x_{1} y_{2}\right) & x_{2} y_{2} & \cdots \\
\vdots & \vdots &
\end{array}\right) \in \operatorname{Sym}(n \times n) .
$$

Given $t \in U$, we can define a unit length vector $e_{1}(t) \in \mathbf{R}^{n}$ in such a way that

$$
g(t)=\left(\sqrt{n^{2}-n}\right)^{-1}\left[(1-n) e_{1}(t) \circ e_{1}(t)+\left(e_{2} \circ e_{2}+\cdots+e_{n} \circ e_{n}\right)\right],
$$

where $e_{2}, \ldots, e_{n}$ are any vectors completing $e_{1}(t)$ to an orthonormal basis for $\mathbf{R}^{n}$. Indeed, we can take $e_{1}(t)$ to be a unit-length vector in the one-dimensional eigenspace for $g(t)$ corresponding to eigenvalue $(1-n) / \sqrt{n^{2}-n}$, an eigenspace which depends smoothly on $g(t)$. 
We will now define the set $R$ of regular points, and if $t_{0} \in R$, we will specify a moving orthonormal frame on a neighborhood of $t_{0}$ : Let $t_{0} \in U$. We begin with $e_{1}(t)$, defined as in the preceding paragraph. If $e_{1}^{\prime}(t)=0$ on some neighborhood $I$ of $t_{0}$, we say that $t_{0} \in R$, and we choose $e_{2}(t), \ldots, e_{n}(t)$ for $t \in I$ so that $\left(e_{1}(t), \ldots, e_{n}(t)\right)$ is orthonormal and $e_{i}^{\prime}(t)=0$. If $e_{1}^{\prime}\left(t_{0}\right)=0$ but $e_{1}^{\prime}(t) \neq 0$ for $t$ arbitrarily near $t_{0}$, we say that $t_{0} \notin R$ and omit it from further consideration. Finally, if $e_{1}^{\prime}\left(t_{0}\right) \neq 0$, we select a unit-length vector $e_{2}(t)$, for $t$ in some neighborhood of $t_{0}$, so that $\left(d e_{1} / d t\right)(t)=\kappa_{1}(t) e_{2}(t)$ on this neighborhood, and we proceed to the next step in the construction of the moving orthonormal frame.

If $\left(e_{1} \wedge e_{2}\right)^{\prime}(t)=0$ on a neighborhood of $t_{0}$, we say that $t_{0} \in R$ and we choose $e_{3}(t), \ldots, e_{n}(t)$ on this neighborhood of $t_{0}$ so that $\left(e_{1}(t), \ldots, e_{n}(t)\right)$ is orthonormal and $e_{i}^{\prime}(t)=0$ for $3 \leqslant i \leqslant n$. If $\left(e_{1} \wedge e_{2}\right)^{\prime}\left(t_{0}\right)=0$ but $\left(e_{1} \wedge e_{2}\right)^{\prime}(t) \neq 0$ for $t$ arbitrarily near $t_{0}$, we say that $t_{0} \notin R$ and omit it from further consideration. Finally, if ( $\left.e_{1} \wedge e_{2}\right)^{\prime}\left(t_{0}\right) \neq 0$, we choose $e_{3}(t)$ for $t$ in some neigborhood of $t_{0}$, so that $e_{3}(t)$ is perpendicular to $e_{1}(t)$ and $e_{2}(t)$, and $d e_{2} / d t=-\kappa_{1} e_{1}+\kappa_{2} e_{3}$, and we proceed to the next step.

Continue in this fashion until either it is determined that $t_{0} \notin R$, or a complete orthonormal frame $\left(e_{1}(t), \ldots, e_{n}(t)\right)$ is constructed on some neighborhood of $t_{0}$. In the latter case, $t_{0} \in R$ and the moving orthonormal frame satisfies the "FrenetSerret formula",

$$
\left(\begin{array}{c}
e_{1}^{\prime} \\
e_{2}^{\prime} \\
e_{3}^{\prime} \\
\vdots \\
e_{n}^{\prime}
\end{array}\right)=\left(\begin{array}{ccccc}
0 & \kappa_{1} & 0 & \cdots & 0 \\
-\kappa_{1} & 0 & \kappa_{2} & \cdots & 0 \\
0 & -\kappa_{2} & 0 & \cdots & \\
\vdots & \vdots & \vdots & & \\
0 & 0 & & &
\end{array}\right)\left(\begin{array}{c}
e_{1} \\
e_{2} \\
e_{3} \\
\vdots \\
e_{n}
\end{array}\right)
$$

where some of the $\kappa_{i}$ 's may be identically zero.

Using these formulae, it is possible to differentiate (8) and obtain quite explicit expressions for the derivatives $g^{(k)}\left(t_{0}\right)$, when $t_{0} \in R$ :

$$
\begin{gathered}
g^{\prime}\left(t_{0}\right)=\left(\sqrt{n^{2}-n}\right)^{-1}\left[-2 n \kappa_{1} e_{1} \circ e_{2}\right]\left(t_{0}\right), \\
g^{\prime \prime}\left(t_{0}\right)=\left(\sqrt{n^{2}-n}\right)^{-1}\left[-2 n \kappa_{1}^{\prime} e_{1} \circ e_{2}-2 n \kappa_{1}^{2} e_{2} \circ e_{2}-2 n \kappa_{1} e_{1} \circ\left(-\kappa_{1} e_{1}+\kappa_{2} e_{3}\right)\right]\left(t_{0}\right),
\end{gathered}
$$

etc. The crucial observation is that $g^{(k)}\left(t_{0}\right)$ is a linear combination of $e_{i}\left(t_{0}\right) \circ e_{j}\left(t_{0}\right)$, with the $e_{n}\left(t_{0}\right) \circ e_{n}\left(t_{0}\right)$ term making its first appearance only when $k$ has reached at least $2 n-2$. Since

$$
\left\langle\left\langle e_{i}\left(t_{0}\right) \circ e_{j}\left(t_{0}\right), e_{n}\left(t_{0}\right) \circ e_{n}\left(t_{0}\right)\right\rangle\right\rangle=0 \quad \text { unless } i=j=n,
$$

we conclude that

$$
1 \leqslant k\left\langle 2 n-3 \Rightarrow\left\langle\left\langle g^{(k)}\left(t_{0}\right), e_{n}\left(t_{0}\right) \circ e_{n}\left(t_{0}\right)\right\rangle\right\rangle=0,\right.
$$

where $\langle\langle\rangle$,$\rangle is the standard inner product on \operatorname{Sym}(n \times n)$ described earlier. 
Now we apply the Leibniz formula,

$$
f^{(k)}=\tau^{(k)} g+k \tau^{(k-1)} g^{\prime}+\cdots+k \tau^{\prime} g^{(k-1)}+\tau g^{(k)},
$$

take the inner product with $e_{n}\left(t_{0}\right) \circ e_{n}\left(t_{0}\right)$, and apply (9) to obtain

$$
\begin{aligned}
\left\langle\left\langle f^{(k)}\left(t_{0}\right), e_{n}\left(t_{0}\right) \circ e_{n}\left(t_{0}\right)\right\rangle\right\rangle & =\tau^{(k)}\left(t_{0}\right)\left\langle\left\langle g\left(t_{0}\right), e_{n}\left(t_{0}\right) \circ e_{n}\left(t_{0}\right)\right\rangle\right\rangle \\
& =\left(\sqrt{n^{2}-n}\right)^{-1} \tau^{(k)}\left(t_{0}\right),
\end{aligned}
$$

which implies that

$$
\left|\tau^{(k)}\left(t_{0}\right)\right| \leqslant \sqrt{n^{2}-n}\left|f^{(k)}\left(t_{0}\right)\right|, \quad \text { for } 0 \leqslant k \leqslant 2 n-3 .
$$

This gives the desired estimate $\left(E_{k}\right)$ when we set $C_{k}=\sqrt{n^{2}-n}$, and hence the Sublemma is proved.

4. Differentiability. To finish the proof of the theorem stated in the introduction, we need to show that the sections $e_{n+1}, e_{n+2}: M \rightarrow N M$ constructed in $\$ 2$, where $N M$ is the normal bundle of the isometric immersion $f: M^{n} \rightarrow E^{n+2}$, are differentiable of class $C^{k}$.

Let us consider the sections $\phi_{1}=\left\langle\alpha, e_{n+1}\right\rangle$ and $\phi_{2}=\left\langle\alpha, e_{n+2}\right\rangle$ of the bundle $L_{s}^{2}(T M)$ of symmetric covariant tensors of rank two on $M . \phi_{1}$ is $C^{k+1}$ because we also have $\phi_{1}=\left\langle\tilde{\alpha}, \tilde{e}_{n+1}\right\rangle$, where $\tilde{\alpha}$ is $C^{k+1}$ and $\tilde{e}_{n+1}$ is $C^{k+2}$, and $\phi_{1}$ is positive definite by our curvature assumption. Hence, $\phi_{1}$ is a new Riemannian metric on $M$; geometrically, it is the pullback of the standard unit curvature metric on $S^{n}$ via the Gauss map of $\tilde{f}: M \rightarrow E^{n+1}$.

It will suffice to work locally. In a neigborhood $U$ of a given point $p \in M$, we choose vector fields $\left(e_{1}, \ldots, e_{n}\right)$ which are orthonormal with respect to $\phi_{1}$. Set $H=\sum_{i=1}^{n} \alpha\left(e_{i}, e_{i}\right)$, a nonvanishing $C^{k+1}$ section of the normal bundle over $U$, and let $\hat{e}_{n+1}$ be the unit length normal field pointing in the direction of $H$. Finally, let $\hat{e}_{n+2}$ be the normal field obtained from $\hat{e}_{n+1}$ by counterclockwise rotation through $\pi / 2$ in the direction determined by the orientation of the normal bundle. $\hat{e}_{n+1}$ and $\hat{e}_{n+2}$ are $C^{k+1}$ sections of the normal bundle.

We define a function $\theta: U \rightarrow \mathbf{R}$ by requiring that $-\pi / 2<\theta<\pi / 2$ and

$$
\left(\begin{array}{l}
e_{n+1} \\
e_{n+2}
\end{array}\right)=\left(\begin{array}{cc}
\cos \theta & -\sin \theta \\
\sin \theta & \cos \theta
\end{array}\right)\left(\begin{array}{l}
\hat{e}_{n+1} \\
\hat{e}_{n+2}
\end{array}\right) \text {. }
$$

To show that $e_{n+1}$ and $e_{n+2}$ are $C^{k}$, it will suffice to show that $\theta$ is $C^{k}$. Let $\psi_{1}=\left\langle\alpha, \hat{e}_{n+1}\right\rangle$ and $\psi_{2}=\left\langle\alpha, \hat{e}_{n+2}\right\rangle$, so that

$$
\left(\begin{array}{l}
\psi_{1} \\
\psi_{2}
\end{array}\right)=\left(\begin{array}{cc}
\cos \theta & \sin \theta \\
-\sin \theta & \cos \theta
\end{array}\right)\left(\begin{array}{l}
\phi_{1} \\
\phi_{2}
\end{array}\right) .
$$

We will need to use the $C^{k+1}$ inner product $\langle\langle\rangle$,$\rangle defined on sections \phi, \psi$ of $L_{s}^{2}(T M) \mid U$ by

$$
\langle\langle\phi, \psi\rangle\rangle=\sum_{i, j=1}^{n} \phi\left(e_{i}, e_{j}\right) \psi\left(e_{i}, e_{j}\right),
$$


where $\left(e_{1}, \ldots, e_{n}\right)$ are orthonormal with respect to $\phi_{1}$. In terms of this inner product, $\left\langle\left\langle\phi_{1}, \phi_{1}\right\rangle\right\rangle=n$ and $\left\langle\left\langle\psi_{2}, \phi_{1}\right\rangle\right\rangle=0$, and hence

$$
\begin{gathered}
0=\left\langle\left\langle-\sin \theta \phi_{1}+\cos \theta \phi_{2}, \phi_{1}\right\rangle\right\rangle=-n \sin \theta+\cos \theta\left\langle\left\langle\phi_{2}, \phi_{1}\right\rangle\right\rangle \\
\Rightarrow\left\langle\left\langle\phi_{1}, \phi_{2}\right\rangle\right\rangle=n \tan \theta, \\
\left\langle\left\langle\phi_{1}, \psi_{1}\right\rangle\right\rangle=\cos \theta\left\langle\left\langle\phi_{1}, \phi_{1}\right\rangle\right\rangle+\sin \theta\left\langle\left\langle\phi_{2}, \phi_{1}\right\rangle\right\rangle \\
=n \cos \theta+n \sin \theta \tan \theta=n \sec \theta \\
\Rightarrow \sec \theta=(1 / n)\left\langle\left\langle\phi_{1}, \psi_{1}\right\rangle\right\rangle .
\end{gathered}
$$

Since $\phi_{1}$ and $\psi_{1}$ are $C^{k+1}$, so is $\sec \theta$. Thus $\cos \theta$ is $C^{k+1}$ and $\theta$ itself is $C^{k+1}$ except possibly at points where $\theta=0$.

These points where $\theta=0$ are the umbilic points mentioned in the introduction.

Again using our $\phi_{1}$-orthonormal frame $\left(e_{1}, \ldots, e_{n}\right)$, we define a $C^{k+1}$ map $F$ : $U \rightarrow C V$, where $C V$ is the cone on the Veronese manifold studied in $\S 3$, by

$$
F(p)=\text { the symmetric matrix }\left(h_{i j}\right), \text { where } h_{i j}=\psi_{2}\left(e_{i}(p), e_{j}(p)\right) \text {. }
$$

It follows from (10) that

$$
F=-\sin \theta I+(\text { matrix of rank } \leqslant 1),
$$

where $I$ is the identity matrix, and it follows easily from the definition of $\tau$ in $\S 3$ that

$$
\sin \theta=-\left(\sqrt{n^{2}-n}\right)^{-1} \tau \circ F .
$$

Thus it remains only to show that $\tau \circ F$ is $C^{k}$ on some neighborhood of the given point $p$. If $\eta: M \rightarrow[0,1]$ is a smooth function which is identically one on a neighborhood of $p$ and has compact support contained in the open set $U$ considered above, it will suffice to show that $\eta(\tau \circ F)$ is $C^{k}$. We can assume that $\sigma=\left(x^{1}, \ldots, x^{n}\right)$ is a local coordinate system defined on $U$ and extend $(\eta(\tau \circ F)) \circ \sigma^{-1}$ to $\mathbf{R}^{n}$ by setting it equal to zero outside $\sigma(U)$. By abus de langage, we will denote this extended function by $\eta(\tau \circ F) ; \eta(\tau \circ F)$ will henceforth be regarded as a function with compact support defined on $\mathbf{R}^{n}$.

Now it follows immediately from the Cone Lemma of $\$ 3$ that the classical partial derivatives $\left(\partial / \partial x^{i}\right)^{j}(\eta(\tau \circ F))$ exist at every point of $U$ and are bounded, for $1 \leqslant i \leqslant n, 0 \leqslant j \leqslant k+1$. (It is here that the assumption $k \leqslant 2 n-4$ is needed.) Note that the restriction of $\left(\partial / \partial x^{i}\right)^{j} \eta(\tau \circ F)$ to any coordinate line parallel to the $x^{i}$-axis is continuous. If $\phi$ is any $C^{\infty}$ function with compact support in $U$, we can apply Fubini's theorem and integrate by parts to obtain

$$
\begin{aligned}
\int_{U}\left(\partial / \partial x^{i}\right)^{j}(\eta(\tau \circ F)) \phi d x^{1} \cdots d x^{n} \\
\quad=(-1)^{j} \int_{U} \eta(\tau \circ F)\left(\partial / \partial x^{i}\right)^{j} \phi d x^{1} \cdots d x^{n},
\end{aligned}
$$

and hence the classical partial derivatives are also distributional derivatives. In particular, we see that the distributional derivatives

$$
\left(\partial / \partial x^{i}\right)^{j}(\eta(\tau \circ F)) \in L^{p} \text { for all } p, 1 \leqslant i \leqslant n, 0 \leqslant j \leqslant k+1 .
$$


If we were satisfied with a loss of about $n / 2$ derivatives, we could apply Theorem 7.25 (the $L^{2}$ Sobolev imbedding theorem) of Rudin [R]; according to this theorem.

$$
\left(\partial / \partial x^{i}\right)^{j}(\eta(\tau \circ F)) \in L^{2} \text { for } 1 \leqslant i \leqslant n, 0 \leqslant j \leqslant k+1 \Rightarrow \eta(\tau \circ F) \in C^{r},
$$

whenever $r$ is a nonnegative integer satisfying the inequality $r+n / 2<k+1$. Thus $\tau \circ F$ is $C^{r}$ in a neighborhood of $p$.

However, by means of $L^{p}$ regularity theory for elliptic operators, we can do somewhat better. Consider the elliptic operator

$$
\begin{aligned}
L: & \left\{C^{\infty} \text { real-valued functions on } \mathbf{R}^{n}\right\} \\
& \rightarrow\left\{n \text {-tuples of } C^{\infty} \text { real-valued functions on } \mathbf{R}^{n}\right\},
\end{aligned}
$$

defined by

$$
L u=\left(\left(\partial / \partial x^{1}\right)^{k+1} u, \ldots,\left(\partial / \partial x^{n}\right)^{k+1} u\right) .
$$

It follows immediately from (12) that $L(\eta(\tau \circ F)) \in L^{p}$ for all $p$ such that $1<p<$ $\infty$. Hence, the $L^{p}$ regularity theorem for overdetermined elliptic systems (Theorem 7.1 of [A]) implies that $\eta(\tau \circ F) \in L_{k+1}^{p}$, where $L_{k+1}^{p}$ is the Sobolev space of functions on $\mathbf{R}^{n}$ whose distributional derivatives of order $\leqslant k+1$ belong to $L^{p}$. If $p$ is sufficiently large, the Sobolev imbedding theorem implies that $L_{k+1}^{p} \subseteq C^{k}$; we thus conclude that $\eta(\tau \circ F) \in C^{k}$.

It now follows from (11) that $\sin \theta$ is $C^{k}$, and hence the Theorem stated in the introduction is completely proven.

\section{REFERENCES}

[A] S. Agmon, The $L_{p}$ approach to the Dirichlet problem, Ann. Scuola Norm. Sup. Pisa 13 (1959), 405-448.

[BC] R. L. Bishop and R. J. Crittenden, Geometry of manifolds, Academic Press, New York, 1964.

[H1] W. Henke, Riemannsche Mannigfaltigkeiten konstanter positiver Krümmung in euklidischen Räumen der Kodimension 2, Math. Ann. 193 (1971), 265-278.

[H2] _ Über die isometrischer Fortsetzbarkeit isometrischer Immersionen der Standard-m-Sphäre $S^{m}\left(\subset \mathbf{R}^{m+1}\right)$ in $\mathbf{R}^{m+2}$, Math. Ann. 219 (1976), 261-276.

[M] J. D. Moore, Conformally flat submanifolds of Euclidean space, Math. Ann. 225 (1977), 89-97.

[R] W. Rudin, Functional analysis, McGraw-Hill, New York, 1973.

[W] L. Whitt, Isometric homotopy and codimension two isometric immersions of the $n$-sphere into Euclidean space, J. Differential Geometry 14 (1979), 295-302.

Department of Mathematics, University of California, Santa Barbara, California 93106 